\title{
Ultrasound elastography of the supraspinatus tendon guided by US-MRI virtual navigation
}

\author{
Jun Liu, Weiwei Zhan*, Mingyang Zhou and Xiaoxiao Zhang \\ Department of Ultrasonic Diagnosis, Rui Jin Hospital, School of Medicine, Shanghai JiaoTong \\ University, Shanghai, China
}

\begin{abstract}
.
BACKGROUND: A virtual navigation system (VNS) can combine two imaging modalities for synchronous observation and can be beneficial for diagnosis and treatment. Ultrasound elastography (UE) can distinguish between soft and hard tissues. However, the application of UE to musculoskeletal structures is rare.

OBJECTIVE: To evaluate UE of the supraspinatus tendon using ultrasound-magnetic resonance imaging (US-MRI) virtual navigation.

METHODS: Sixty patients with an ache in the shoulder were diagnosed with supraspinatus tendon injuries using MRI. USMRI virtual navigation was used to identify lesions in the supraspinatus tendon, and finally US elastography (UE) was performed. Volunteers whose supraspinatus tendons were diagnosed as normal by MRI were also selected for US elastography. The UE scores were then compared between patients and volunteers.

RESULTS: A total of 60 patients were diagnosed with supraspinatus tendon injuries using MRI and forty-two patients classified as Grade I exhibited no obvious abnormality by US. The supraspinatus tendon injury scores were significantly higher in Grade I patients compared with volunteers $(P<0.05)$.

CONCLUSION: US elastography is helpful for the diagnosis of the supraspinatus tendon injuries when used with US-MRI virtual navigation.
\end{abstract}

Keywords: Ultrasound elastography, supraspinatus tendon, virtual navigation

\section{Introduction}

The rotator cuff, which includes the supraspinatus, infraspinatus, small round muscle, subscapular muscles, and tendons, is an important structure in the shoulder that maintains stable biomechanics. Supraspinatus tendon injuries are the most common shoulder injury [1|2]. While we can gain information concerning echogenicity and structure from gray-scale imaging and information relating to vascularity from color Doppler sonography, ultrasonography (US) can be used to examine both muscles and tendons. Rotator-cuff injuries diagnosed by US have been reported widely [3|4], but diffuse and imperceptible lesions in the supraspinatus tendon cannot always be identified using US. Magnetic resonance imaging (MRI) plays an important role in the diagnosis of rotator-cuff injury [5]6]. A virtual navigation system (VNS) can combine two imaging modalities for synchronous observation and can be beneficial for diagnosis and treatment. Ultrasound elastography (UE) can distinguish between the soft and hard

\footnotetext{
${ }^{*}$ Corresponding author: Weiwei Zhan, Department of Ultrasonic Diagnosis, Rui Jin Hospital, School of Medicine, Shanghai JiaoTong University, 197 Ruijin NO. 2 Road, Shanghai 200025, China. Tel.: +86 02164370045 600303; E-mail: shanghairuijin@126.com.
} 
tissues, particularly in the breast or thyroid [7]8], and has also been widely reported. However, the application of UE to musculoskeletal structures is rare [9]. This study was performed to assess the value of UE in supraspinatus tendon injuries, after sonography was performed on the basis of MRI images, to identify lesions with VNS.

\section{Materials and methods}

\subsection{Patients and volunteers}

This study was approved by the hospital Institutional Review Board, and signed informed consent was obtained from both patients and volunteers. Sixty patients presenting with an ache on one shoulder were selected between December 2011 and March 2012. Using MRI, all patients were diagnosed with supraspinatus tendon injuries on one side. Diagnosis was performed according to the Zlatkins rotator cuff injury classification standard, in which patients are classified in three grades based on the appearance, local signal strength, and continuity of the tendon (Grade I: tendon appearance and continuity are normal, tendon interior exhibits anomalous signals; Grade II: tendinosis or degeneration, tendon becomes thinner or irregular in appearance. Grade III: rotator cuff tear) [10].

Volunteers with normal supraspinatus tendons as diagnosed by MRI were selected for comparison with Grade 1 patients.

\section{Equipment and software}

This study was performed using a sonography scanner incorporating a 12-MHz linear-array probe, a magnetic field generator, a magnetic sensor, and a workstation with built-in VNS software and quasistatic UE software (Mylab 90, Esaote SpA, Italy). The sequences and parameters of MRI (Signa HDx 3.0 T, GE, USA) were as follows: Sequence 1: Ocor T1, time of echo (TE) $12.8 \mathrm{~ms}$, time of repetition (TR) $660 \mathrm{~ms}$, field of view (FOV) $18 \mathrm{~cm}$, thickness: $4.0 \mathrm{~mm}$, spacing: $1.0 \mathrm{~mm}$, Matrix $320 \times 192$, NEX 2.00. Sequence 2: OSag T2, TE $85 \mathrm{~ms}$, TR $2800 \mathrm{~ms}$, FOV $16 \mathrm{~cm}$, thickness: $4.0 \mathrm{~mm}$, spacing: $1.0 \mathrm{~mm}$, Matrix $320 \times 224$, NEX 2.00. Sequence 3: OAx fs T2, TE $72 \mathrm{ms,} \mathrm{TR} 2800 \mathrm{~ms}$, FOV $16 \mathrm{~cm}$, thickness: $5.0 \mathrm{~mm}$, spacing: $1.0 \mathrm{~mm}$, Matrix $288 \times 160$, NEX 4.00. Ocor fs T2, TE $75 \mathrm{~ms}$, TR $2800 \mathrm{~ms}$, FOV $18 \mathrm{~cm}$, thickness: $4.0 \mathrm{~mm}$, spacing: $1.0 \mathrm{~mm}$, Matrix $320 \times 192$, NEX 4.00 .

Before initiation of the VNS, MRI volume data were transferred to the VNS with Digital Imaging and Communications in Medicine (DICOM) data. The VNS could display the sonography and MRI images simultaneously, and the two imaging modalities could be changed simultaneously.

\subsection{Experimental procedure}

First, patients with a shoulder ache were examined by MRI, and then sonography images were captured using the MRI images as guidance to identify lesions using VNS. During US, the same prone patient position was adopted as during the MRI procedure. VNS exited after identification of lesions, and then UE was performed in the US mode. Care was taken to ensure that the region of interest (ROI) in the UE mode included the lesions. Images were frozen and acquired when the UE images became stable.

Second, UE was performed according to the same procedure in volunteers whose supraspinatus tendons were diagnosed as normal by MRI.

Finally, the UE images of all patients and volunteers were assessed by two professional US doctors. 


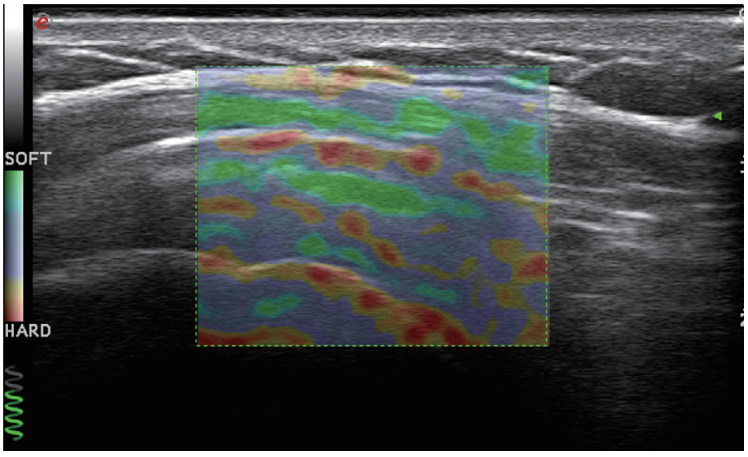

Fig. 1. Elastography image, score $=2$.

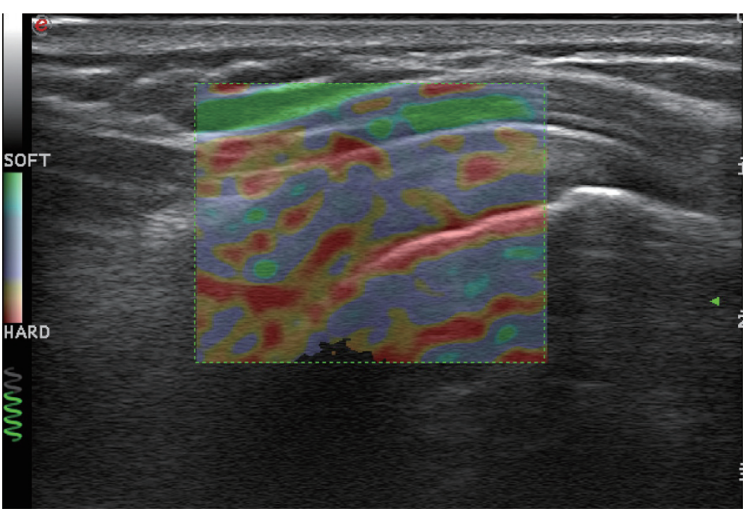

Fig. 3. Elastography image, score $=4$.

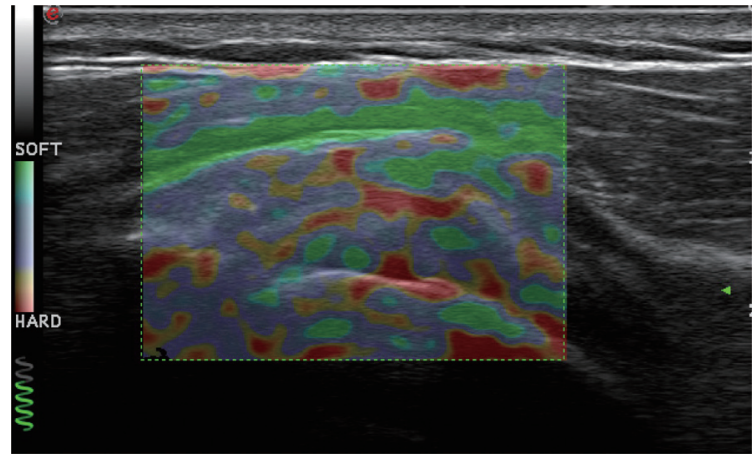

Fig. 2. Elastography image, score $=3$.

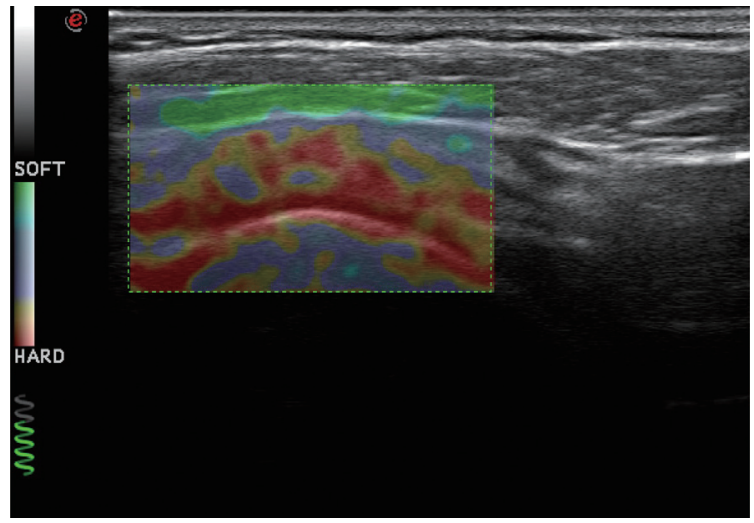

Fig. 4. Elastography image, score $=5$.

In a similar way to the application of UE in the breast [11] and according to the colors of the UE images, the UE images were divided into five grades: Score 1: More than $90 \%$ green with the remainder purple or red; Score 2: Approximately 50-90\% green, $<10 \%$ red, and the remainder purple; Score 3: $>50 \%$ purple with the remainder green and red, or $>90 \%$ green mixed with red; Score 4: Approximately 50$90 \%$ red and $<10 \%$ green, with the remainder purple; Score $5:>90 \%$ green with the remainder purple or red [11] (Figs 1 to 4 ).

\subsection{Statistical analysis}

Grade I patients were selected for the final statistical analysis, with patients in Grade II and Grade III excluded from the analysis post hoc. Rank tests and inspection were used to compare UE scores between Grade I patients and volunteers. $P<0.05$ was considered statistically significant for all tests.

\section{Results}

Sixty patients classified with rotator cuff injury and 42 healthy volunteers with normal supraspinatus tendons were included in this study. Of the 60 patients, 22 were male and 38 were female, and the average age was 56.8 years (range, 38 years- 75 years). Fifty-five patients had a right rotator cuff injury and five 


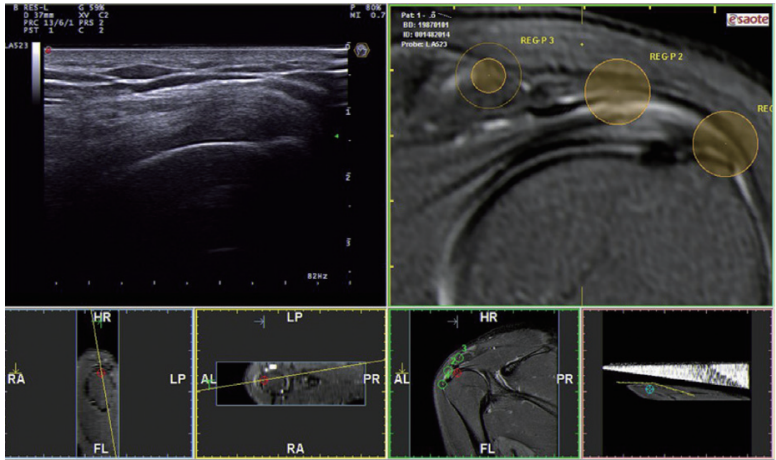

(a)

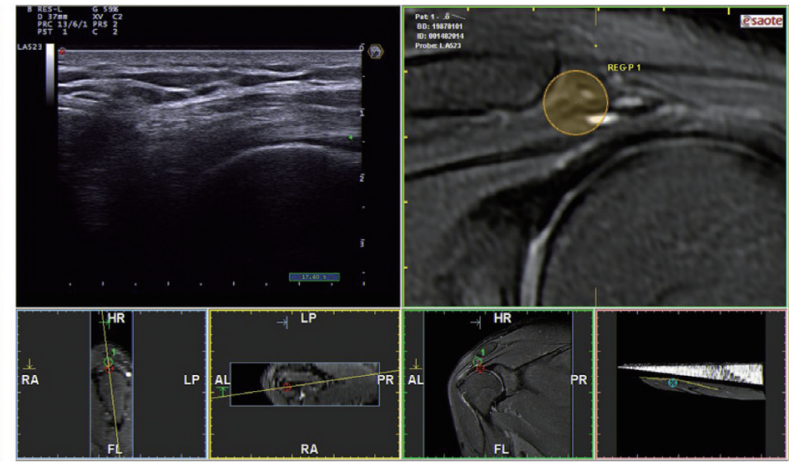

(b)

Fig. 5. Sonography images guided according to the MRI images to identify lesions using VNS.

Table 1

Comparison of the scores between the patients in Grade I and the volunteers

\begin{tabular}{lccrrr}
\hline \multicolumn{5}{c}{ Scores } & \\
\hline Groups & 1 & 2 & 3 & 4 & 5 \\
Grade I & 0 & 0 & 0 & 19 & 23 \\
Volunteers & 0 & 3 & 32 & 7 & 0 \\
$P<0.05$ & & & & & \\
\hline
\end{tabular}

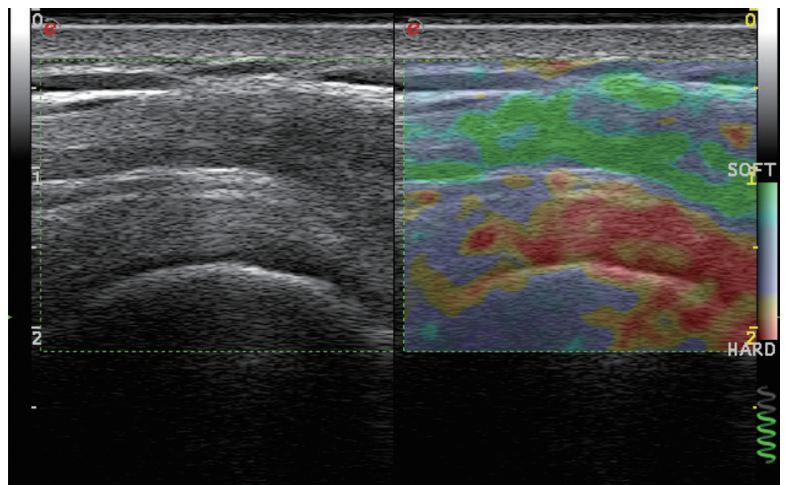

Fig. 6. Elastography images of supraspinatus tendons, score $=$ 5 .

had an injury on the left side. According to the Zlatkins rotator cuff injury classification standard, 42 (70.00\%) patients were classified as Grade I, nine (15.00\%) patients were classified as Grade II, and nine $(15.00 \%)$ were classified as Grade III. Grey-scale US showed no obvious abnormality in the 42 Grade I patients. All sonography images could be guided by the MRI images to identify the lesions with VNS (Figs 5(a) and (b)).

US-MRI virtual navigation among Grade I patients revealed $19(45.24 \%)$ supraspinatus tendons with a score of 4 and $23(54.76 \%)$ with a score of 5 . Among healthy volunteers, three (7.14\%) supraspinatus tendons scored 2, $32(76.19 \%)$ scored 3, and seven (16.67\%) scored 4. There were no score of 1, 2 and 3 in Grade I patients and no score of 1 and 5 in healthy volunteers.

The supraspinatus tendon scores of all patients and volunteers are shown in Table 1 and Fig. 6 . The scores of Grade I patients were significantly higher than those of the volunteers $(P<0.05)$.

\section{Discussion}

In 1996, Oshio and Shinmoto [12] were the first to report the application of an integrated system incorporating computer tomography (CT) and sonographic images. Using VNS, the two imaging modalities of MRI and sonography can be shown synchronously in real time, side-by-side. This technique was 
used to guide sonography images to identify lesions according to the MRI images in our study. As a panoramic image of the supraspinatus tendon can be shown in an oblique coronal MRI, oblique coronal MRI images were used for guiding the process of US-MRI virtual navigation. Because a number of images were captured at different degrees of injury in different MRI sequence images, we selected the most representative images in our study [13-15]. The process of US-MRI virtual navigation provides a good platform for subsequent UE.

In our study, grey-scale US did not show any obvious abnormality in the 42 Grade I patients because the supraspinatus tendon did not rupture in the grey-scale US images. However, the supraspinatus tendon appeared abnormal in the MRI images because of the use of the two different imaging modalities, US and MRI. Some lesions in the supraspinatus tendon in the Grade I group could not be seen clearly in greyscale US because of the similar acoustic impedance between the lesions and the surrounding tissues. Because the radiographic imaging principle of MRI is different from that of US, the lesions could be identified in the MRI images but not in the same position in the grey-scale US images.

Unfortunately, due to the inconvenience and expense of MRI, this is not a suitable method for longterm follow-up. In contrast, UE can be used for long-term follow-up because of its convenience and repeatability. UE can be also used dynamically to observe the changes in lesions in the supraspinatus tendon, including hardness and range. Furthermore, UE may be better than MRI in assessing tendon injury. Following one MRI examination only, patients' MRI images can be stored in VNS, and made available for guidance at any time when required.

The pathological process of tendon injury can be divided into three distinct periods; the edema bleeding, chronic tendinosis, and tendon rupture periods [2]. During the injury process, the body's repair mechanisms are activated simultaneously. Hyperplastic fiber cells secrete collagen fiber and form a scar, increasing the hardness of the tendon and increasing UE scores. In our study, not all of the supraspinatus tendon elastography images were a consistent red because the three pathological periods of the tendons did not occur in the same tendon one after the other. Edema bleeding period, chronic tendinitis period and tendon rupture period can occur in a same tendon at same time. In the edema bleeding period, the tendons did not appear fibrotic and were still in a relatively soft state. UE images showed red mixed with purple, with the appearance even of sheets of green. In the chronic tendinitis period, the tendons appeared fibrotic with the formation of scar tissues, meaning that a red color suggesting contact with hardness was observed in the UE images. In the tendon rupture period, the broken ends of the fractured tendon formed a crack into which synovial fluid from the articulatory antrum flowed, meaning that a green color showing contact with softness was observed in this region in the UE images. Because the lesions in the Grade II and III groups can be seen in grey-scale US images, and the scores can be reduced in the Grade III group because of the synovial fluid in the crack, we focused the UE studies on the Grade I group without tendon rupture. The UE images of the supraspinatus tendon injuries in the Grade I group focused on scores 4-5, while those in the volunteer groups focused on score 3. Most patients in the Grade I group could be still in the chronic tendinitis period, and their scores were statistically significant compared with volunteers $(P<0.05)$.

The statistical results are affected if the scores of the patients in the Grade II and III groups are included the final statistical analysis. If the statistical results include the scores in the Grade II and III groups, particularly the Grade III group, this reduces the total score across the patients. The flow of synovial fluid in the articulatory antrum into the crack means that the scores of the UE images in the Grade III group may be underestimated. Patients in the Grade II and Grade III groups were therefore post hoc excluded the final statistical analysis. Because the lesions in the Grade II and III groups can be shown in grey-scale US images, even if these patients were not included the final statistical analysis, the 
lesions could not be omitted. Therefore, we excluded these patients in the Grade II and III groups in the final statistical analysis.

In conclusion, with VNS, sonography images can be guided by MRI images to identify lesions that are not observed using grey-scale US. UE may therefore be useful for supraspinatus tendon injury in patients with a shoulder ache.

\section{Acknowledgements}

This study was financially supported by the project grant from Shanghai Jiao Tong University. No. YG2012ZD06. The authors are grateful to all study participants.

\section{References}

[1] A.M. Halder, E. Itoi and K.N. An, Anatomy and biomechanics of the shoulder, Orthop Clin North Am 31 (2000), 159176.

[2] J.Y. Ko, C.C. Huang, W.J. Chen, C.E. Chen, S.H. Chen and C.J. Wang, Pathogenesis of partial tear of the rotator cuff: a clinical and pathologic study, J Shoulder Elbow Surg 15 (2006), 271-278.

[3] A. Papatheodorou, P. Ellinas, F. Takis, A. Tsanis, I. Maris and N. Batakis, US of the shoulder: rotator cuff and non-rotator cuff disorders, Radiographics 26 (2006), e23.

[4] J.P. Iannotti, J. Ciccone, D.D. Buss, J.L. Visotsky, E. Mascha, K. Cotman and N.M. Rawool, Accuracy of office-based ultrasonography of the shoulder for the diagnosis of rotator cuff tears, J Bone Joint Surg Am 87 (2005), 1305-1311.

[5] E.G. McNally and J.L. Rees, Imaging in shoulder disorders, Skeletal Radiol 36 (2007), 1013-1016.

[6] A.B. Wolff, P. Sethi, K.M. Sutton, A.S. Covey, D.P. Magit and M. Medvecky, Partial-thickness rotator cuff tears, J Am Acad Orthop Surg 14 (2006), 715-725.

[7] K.M. Hiltawsky, M. Kruger, C. Starke, L. Heuser, H. Ermert and A. Jensen, Freehand ultrasound elastography of breast lesions: clinical results, Ultrasound Med Biol 27 (2001), 1461-1469.

[8] W. Khaled, S. Reichling, O.T. Bruhns and H. Ermert, Ultrasonic strain imaging and reconstructive elastography for biological tissue, Ultrasonics 44 Suppl 1 (2006), e199-202.

[9] P. Lalitha, M. Reddy and K.J. Reddy, Musculoskeletal applications of elastography: a pictorial essay of our initial experience, Korean J Radiol 12 (2011), 365-375.

[10] L.L. Seeger, Magnetic resonance imaging of the shoulder, Clin Orthop Relat Res (1989), 48-59.

[11] A. Itoh, E. Ueno, E. Tohno, H. Kamma, H. Takahashi, T. Shiina, M. Yamakawa and T. Matsumura, Comparison between ultrasonic elastogram and histologic findings in breast diseases, The Seventh Congress of Asian Federation of Societies for Ultrasound in Medicine and Biology (AFSUMB 2004) (2004), 330.

[12] K. Oshio and H. Shinmoto, Simulation of US imaging by using a 3D data set, Radiology 201 (1996), 517.

[13] W.E. Palmer, P.L. Caslowitz and F.S. Chew, MR arthrography of the shoulder: normal intraarticular structures and common abnormalities, AJR Am J Roentgenol 164 (1995), 141-146.

[14] S.F. Quinn, R.C. Sheley, T.A. Demlow and J. Szumowski, Rotator cuff tendon tears: evaluation with fat-suppressed MR imaging with arthroscopic correlation in 100 patients, Radiology 195 (1995), 497-500.

[15] A.M. Evancho, R.G. Stiles, W.A. Fajman, S.P. Flower, T. Macha, M.C. Brunner and L. Fleming, MR imaging diagnosis of rotator cuff tears, AJR Am J Roentgenol 151 (1988), 751-754. 